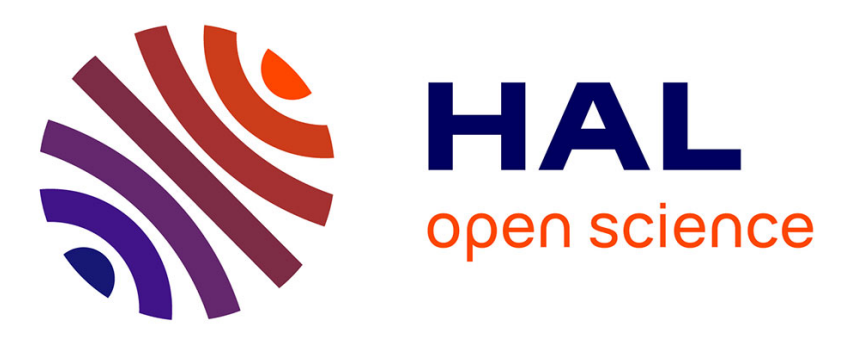

\title{
An On-Line Real-Time Monitoring Method for Locating Natural Triggered Events on Medium Voltage Power Grids
}

\author{
Moussa Kafal, Nicolas Gregis, Jaume Benoit, Nicolas Ravot
}

\section{To cite this version:}

Moussa Kafal, Nicolas Gregis, Jaume Benoit, Nicolas Ravot. An On-Line Real-Time Monitoring Method for Locating Natural Triggered Events on Medium Voltage Power Grids. URSI GASS 2020

- XXXIII General Assembly and Scientific Symposium, International Union of Radio Science (Union Radio Scientifique Internationale-URSI), Aug 2020, Rome, Italy. pp.PID6347245. cea-03078799

\section{HAL Id: cea-03078799 \\ https://hal-cea.archives-ouvertes.fr/cea-03078799}

Submitted on 16 Dec 2020

HAL is a multi-disciplinary open access archive for the deposit and dissemination of scientific research documents, whether they are published or not. The documents may come from teaching and research institutions in France or abroad, or from public or private research centers.
L'archive ouverte pluridisciplinaire HAL, est destinée au dépôt et à la diffusion de documents scientifiques de niveau recherche, publiés ou non, émanant des établissements d'enseignement et de recherche français ou étrangers, des laboratoires publics ou privés. 


\title{
An On-Line Real-Time Monitoring Method for Locating Natural Triggered Events on Medium Voltage Power Grids
}

\author{
Moussa kafal $^{(1)}$, Nicolas Gregis ${ }^{(1)}$, Jaume Benoit ${ }^{(1)}$, and Nicolas Ravot ${ }^{(1)}$ \\ (1) CEA, LIST, Laboratoire de Fiabilité et Intégration Capteurs, 91191 Gif-sur-Yvette, France
}

\begin{abstract}
Time reversal (TR) has been recently adapted with success to locate faults in power grids, thanks to its location accuracy and robustness against parameters uncertainties. FasTR algorithm, a post-processing approach based on the tenets of TR, has shown promising results in locating disturbances along transmission line networks. This paper presents the outcome of tests performed on a $2.6 \mathrm{~km}$ energized three-phase $20 \mathrm{kV}$ buried pilot network. The tests involve single-phase-to-ground fault occurrences manually triggered, and a 3-month real-time continuous monitoring while the network is in normal operation. The fault location task is performed by a system composed four components: distributed high-speed measurement devices, accurate time synchronization, a communication tool between sensors and the FasTR post-processing algorithm. The obtained results demonstrated the capacity of an on-the-shelf portable system to make an on-line accurate detection of transient faults in a complex network.
\end{abstract}

\section{Introduction}

The dependency on electricity has grown significantly in modern times and the continuous availability of electric energy has become imperative to uphold the basic functions of the twenty-first-century industrialized society. This has entailed a growing interest in increasing the reliability of power grids where medium voltage (MV) cables form an integral part. With a view to increasing security of supply and due to the continued decrease of cost of underground solutions, MV networks have been widely deployed in fully under-grounded electricity distribution network across Europe [1]. However, as any cable they are prone to deterioration and aging thus leading to the occurrence of faults ending to outages and power interruptions.

In effect, much work has been invested in new technological solutions for fault location on power grids. Nevertheless, underground MV networks implicated higher maintenance complexity due to their hard-access situation. On the other hand, non-destructive localization methods among those based on traveling waves facilitated fault location and reduced costs $[2,3]$. The major drawbacks of such methods is that they require an assessment between the number of observation points versus the number of possible multiple fault-location solutions and require large bandwidth measuring systems.

A recent proposal for fault location is electromagnetic time reversal (EMTR) [4], which is inspired from the idea of time reversal (TR) focusing [5]. It has proven an ability of locating faults from their emitted transient signals recorded from a single probe in MV networks through full-scale experimental validations [6]. However, its performance has been shown to rely on a well-defined network topology combined with precision hardware. Besides, since it deploys one sensor on a single observation point, it necessitated the presence of adapters to fix the loads on the network extremities. This was meant to ensure the occurrence of rebounds or multiple reflections at these points and thus enable a better network coverage. Particularly, this was necessary when dealing with networks composed of junctions and branches with long cables.

Accordingly, we will present in this paper a post-processing method, referred to as FasTR, based on the tenets of TR theory capable of bypassing the aforementioned problems. FasTR is shown to extend the possibilities of diagnosing MV networks on a larger scale by placing sensors on wellchosen nodes of the network for continuous monitoring . By using an accurate shared time synchronization system between sensors, transient signals can be recorded with precision then processed on-the-fly with the FasTR algorithm described in this paper.

A comprehensive on-the-shelf portable system developed by Nexans company in collaboration with CEATech is introduced. It demonstrates the ability to capture perturbations or events on a dedicated portion of a given MV network. This system is based on very sensitive and precise acquisition boards (signal amplitude versus time) which can be located at different points of the monitored network. Thanks to the FasTR post-processing algorithm of the collected data, an accurate localization of relevant events can be made. Due to the fact that each acquisition board shares a clock reference based on a GPS antenna, the localization accuracy is within tens of meters.

The system is implemented on an energized $2.6 \mathrm{~km} \mathrm{MV} \mathrm{un-}$ derground power supply system in Switzerland where two sets of full-scale field tests were performed. The first checks 
the system's feasibility to detect manually triggered shortcircuited phase to ground faults. While the second set of tests continuously monitors the network over a period of 3 months to validate the system's performance against naturally triggered events. To the best of the Authors knowledge, this is the first time that such a fault location technique is validated through live long period monitoring.

\section{The Fault location Approach}

Considering a MV network under test (NUT), the occurrence of a fault is associated by high-frequency electromagnetic transients that outbreak and traverse the NUT. These are recorded by high-precision synchronized sensors coupled at distant points of the NUT which are capable of timestamping their moment of arrival. The recorded signals affiliated with their timestamps are then transferred through the communication network to a server where the FasTR algorithm is applied to extract the fault's location.

\subsection{FasTR Algorithm}

FasTR is a variant of TR methods which allows a faster fault localization with a limited required knowledge of the NUT and a moderate need for computing resources. It follows a similar analogy of the EMTR where it employs the already recorded signals, calculates their time-reversed version and estimates a function representative of the energy propagated in the network. But rather than calculating it for a highly refined mesh along time and distance as done with EMTR, FasTR algorithm calculates it for one singular point in the network. Thanks to the advances in optimization methods, the position of the maximum energy and accordingly the fault's location is inferred in few steps. In fact, the construction of the energy function only relies on the time-reversed signals and the topology of the NUT. Particularly, more precise results are accompanied with detailed information of the network. However, a 1 meter fault location accuracy can be still achieved with just the connection matrix and the branch lengths. The FasTR post-processing is performed within less than 1 minute on a standard stateof-art computer. It is noteworthy that the computation time is directly influenced by the precision, where increasing the accuracy consumes more time and vice-versa. Fig. 1 illustrates the flowchart of FasTR algorithm.

\subsection{Full Scale Experimental Validation}

On November 2018, a series of field experiments were performed on an energized three-phase $20 \mathrm{kV}$ buried network in Switzerland. The pilot network was composed of three branches as shown in Fig. 2 with a total length of $2646 \mathrm{~m}$ and easily accessible extremities. Noteworthy, the considered NUT is a section of a bigger underground radial MV distribution network. It possesses built-in protection systems necessary to avoid damaging private properties when generating a transient fault. Three sensors (A,B, and C) were connected at three different locations on the NUT

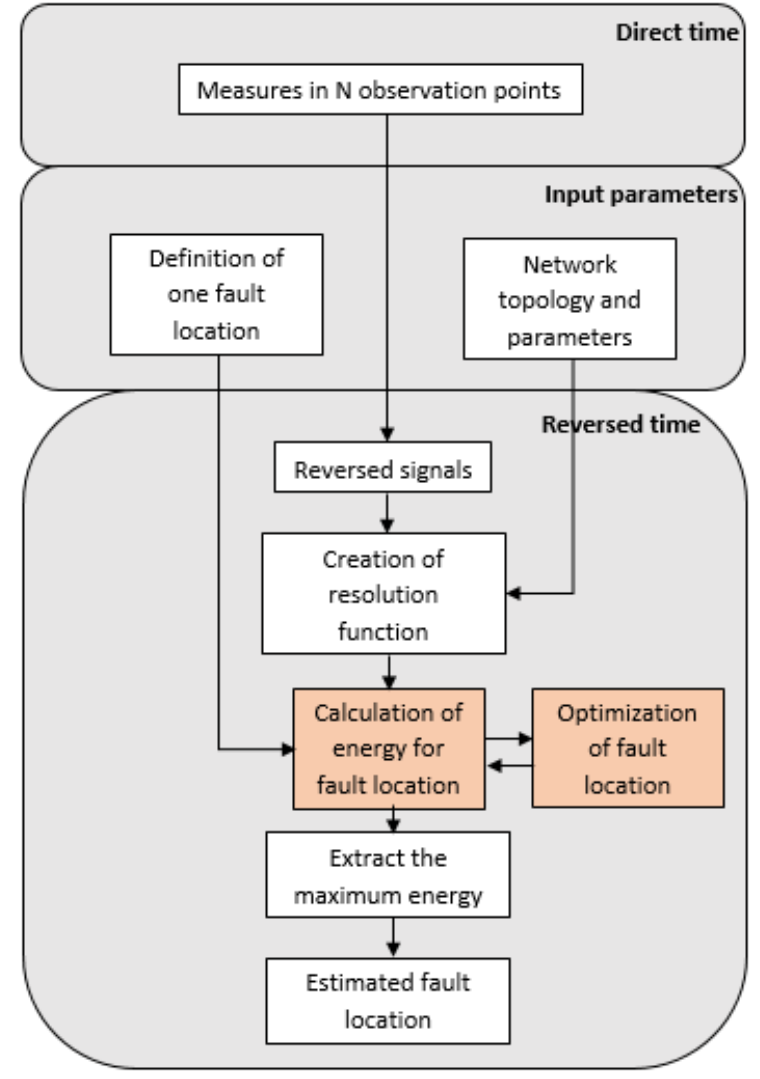

Figure 1. The flowchart of the proposed FasTR algorithm.

through inductive couplers installed on the screen around a phase of the network. These ensured a protection from the low frequency voltage and gave great coupling factors in the $300 \mathrm{kHz}$ to $5 \mathrm{MHz}$ frequency band.

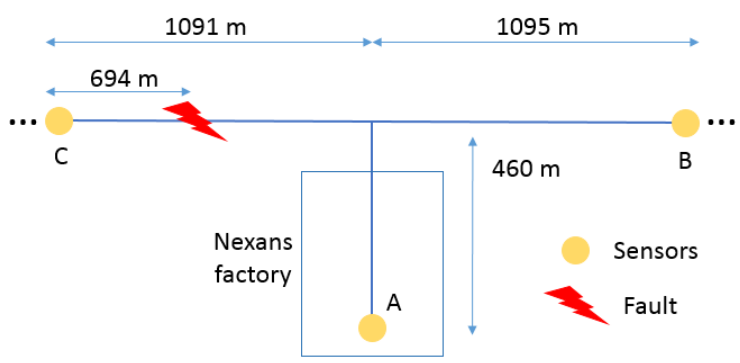

Figure 2. NUT topology with sensors B and C at locations of the network which extends to other parts not covered by the system.

The sensors consisted of National Instrument racks including a PXIe-5122 oscilloscope with a sample rate of $100 \mathrm{MSamples} / \mathrm{s}$ and 14 bit resolution, a PXI-6683H GPS transceiver and PXIe-6674T clocking board which integrates an Oven Controlled X-tal Oscillator (OCXO). The latter device ensured a localization error in the range of two meters. A 4G network guaranteed good communication between sensors and the central server.

A full-scale experimental validation was first performed to test the proposed method's performance. In effect, faults 
were manually generated by an industrial circuit breaker installed $696 \mathrm{~m}$ away from Sensor C that connects one of the 3 phases of the NUT to the ground for $1 \mathrm{~s}$. This created high frequency transients which propagated towards all the extremities of the network and were recorded by the oscilloscope of each sensor. Data was then send to the server where they were processed with the FasTR algorithm.

Although, sensor A wasn't able to detect any signal during the tests, the proposed method correctly localized the branch and phase where faults were generated. Particularly, this means that FasTR can still operate with incomplete data. On the other hand, the accuracy of the system was limited to a fixed error of $55.5 \mathrm{~m}$. For instance, this can be returned to the rough estimate of the propagation speed where an error of $1 \%$ in the wave celerity can lead to a position error of $25 \mathrm{~m}$. However, integrating a precise calibration method can greatly improve the accuracy. Nevertheless, such a result is still satisfying for locating faultoriginating transient signals on power grids.

The promising results obtained motivated a real-time monitoring of the on-line network against "naturally" triggered events by the network or its users. Accordingly, a listening campaign covering 3 months was realized where the already installed system was kept while the circuit breaker was removed. The recorded data were regularly sent from the sensors to the server to be post-processed by the FasTR algorithm. More than 50 events were detected by the monitoring system sorted into three categories (type 1,2, and 3 ) based on the shape of the recorded signals along with a quick spectral analysis. Figure 3 shows the typical shape for event type 1 which occurred on a regular basis throughout the campaign while types 2 and 3 occurred less frequently.

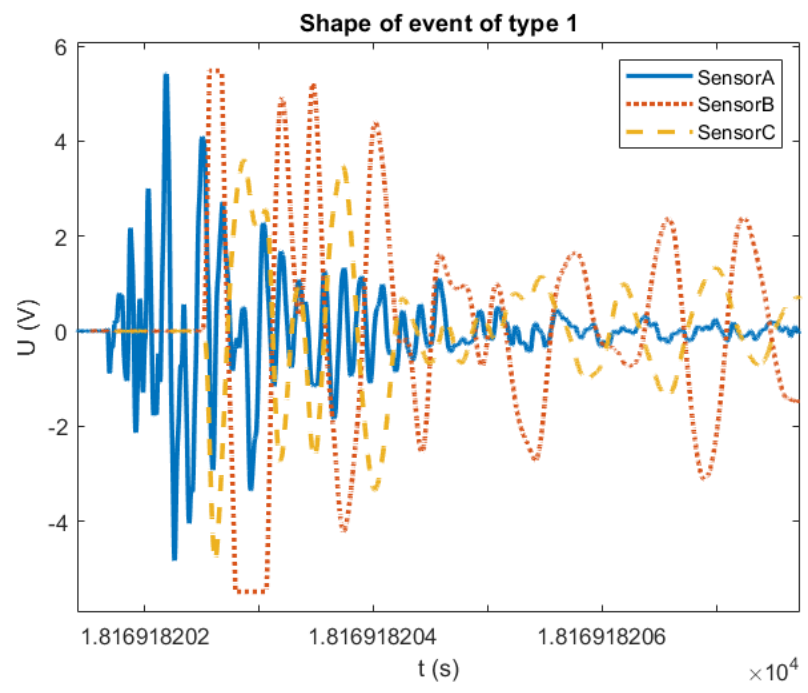

Figure 3. Type 1 fault event recorded by the three sensors.

\section{Conclusion}

This paper has demonstrated the capacity of an on-the-shelf portable system to make an on-line detection of transient faults in a complex network. First, a test was performed on a $2.6 \mathrm{~km} \mathrm{MV}$ network in Switzerland by short-circuiting to the ground a phase of a power supply system. The proposed monitoring system successfully detected the transient fault through its distributed sensors, thanks to a high-quality time synchronization combined with the FasTR post-processing. Then a campaign of online measurements were realized for a duration of 3 months. The monitoring system was able to sort them in three categories based on the shape of their signals and FasTR localization showed a consistency for each type of event, thus proving its capability to detect small transient perturbations. Future work is expected to improve the versatility of the system by reinforcing the automation of FasTR treatments, facilitating the installation in MV/LV stations and collecting data. Besides, a performance analysis investigating the effect of the number of sensors deployed on the tested network should be interesting to be investigated.

\section{Acknowledgements}

The authors would like to thank the contributions of Nexans team for their effective participation in performing the field experimentation.

\section{References}

[1] "An Introduction to Medium and Low Voltage Cables in Distribution Networks as support of Smart Grids," in Europacable, Brussels, June 2014.

[2] P. F. Gale, P. A. Crossley, X. Bingyin, G. Yaozhong, B. J. Cory, and J. R. G. Barker, "Fault location based on travelling waves," in Proc. 5th Int. Conf. Develop. Power Syst. Protection, Mar. 1993, pp. 54-59.

[3] A. O. Ibe and B. J. Cory, "A travelling wave-based fault locator for two- and three-terminal networks," in IEEE Trans. Power Del., vol. 1, no. 2, pp. 283-288, Apr. 1986.

[4] R. Razzaghi, G. Lugrin, H. Manesh, C. Romero, M. Paolone, and F. Rachidi, "An efficient method based on the electromagnetic time reversal to locate faults in power networks," in IEEE Trans. Power Del., vol. 28, no. 3, pp. 1663-1673, Jul. 2013.

[5] M. Fink, "Time reversal of ultrasonic fields. I. Basic principles," in IEEE Trans. Ultrason., Ferroelect. Freq. Control, vol. 39, no. 5, pp. 555-566, Sep. 1992.

[6] Z. Wang, S. He, Q. Li, B. Liu, R. Razzaghi, M. Paolone, Y. Xie, M. Rubinstein and F. Rachidi, "A full-scale experimental validation of electromagnetic time reversal applied to locate disturbances in overhead power distribution lines," in IEEE Trans. EMC, vol. 60, no. 5, pp. 1562-1570, Feb. 2018. 